\title{
Discussion on Warehousing Management Experiment Teaching Mode Based on Virtual Reality Technology
}

\author{
Duan Lijie \\ Experimental Teaching Center, Hubei University of Economics, Wuhan, Hubei Province; School of Logistics \\ and Engineering Management, Hubei University of Economics, Hubei Wuhan
}

Keywords: Virtual reality technology; warehouse management; experimental teaching.

Summary: Using the characteristics of virtual reality technology, combined with the basic theory of warehouse management, in the experimental teaching link, realize the theoretical demonstration of warehouse management through realistic virtual environment, and build modules such as warehouse organization, order picking, ABC classification, replenishment, shelf storage, etc. The traditional teaching mode can freely combine the warehousing function modules, allowing students to interactively teach the content of logistics practice. Students from traditional passive receiving learning to active research learning can greatly improve the experimental teaching effect.

\section{基于虚拟现实技术的仓储管理实验教学模式探讨}

\author{
段李杰 ${ }^{1}$, 涂敏 ${ }^{2}$ \\ 湖北经济学院实验教学中心, 湖北 武汉; 湖北经济学院物流与工程管理学院, 湖北 武汉
}

关键词：虚拟现实技术；仓储管理；实验教学。

摘要: 利用虚拟现实技术的特点, 结合仓储管理的基础理论, 在实验教学环节中通过逼真的 虚拟环境实现仓储管理理论演示, 构建以仓库组织、订单拣选、ABC 分类、补货、货架存储 等模块, 突破了传统的教学模式, 可以自由组合仓储功能模块, 让学生在物流实践内容进行 交互式教学, 学生由传统的被动学习化为我要学, 学以致用的能力训练, 能大大提高实验教 学效果。

仓储管理是一门综合性和实践性很强的课程, 提升课程的教学质量, 锻炼学生的实践应用 能力, 对于提高物流管理专业人才培养质量具有举足轻重的作用。在日常教学环节, 其授课 内容包括仓库选址和布局、仓储设施和设备管理、仓储作业流程管理、库存控制、仓储成本 管理与绩效评估等五个模块。而利用虚拟现实技术可以对这些模块的核心理论进行演示和重 构, 针对性解决理论教学的“空心化”问题, 有效提高实验教学质量, 培养学生的学习兴趣。

\section{1. 现阶段仓储管理课程教学模式存在的问题}

\section{1 理论教学的占比过大, 实验教学环节比例偏低}

仓储管理教学过程中理论部分比重过大，部分课程内容跟其他相关物流课程重复率高，讲 授教师采用“教材加课件”的方式进行内容传授，这种以“老师为主、学生为辅、依靠课本、灌 输知识”的填鸭式教学模式抹杀了学生的学习兴趣, 忽视了学生在学习过程中的主体地位, 学 习积极性不高, 并且容易导致学生分析解决问题的能力不足, 特别是综合分析能力、谋划组 织能力、信息获取和知识再加工能力偏弱。 


\section{2 专业深度不够, 无法深入引导, 缺乏实操}

仓储管理课程是涉及的物流相关实际操作内容覆盖面广、种类多，实践实验教学难度大， 发展还很不成熟, 教学手段相对简单, 许多实践实验教学内容受限于办学条件也是仅仅流于 形式。而对于具有浓厚的技术与实践色彩的物流专业、尤其是实践性很强的《仓储管理》课 程来说, 仅仅局限于课堂讲授和从有限的课本上获取知识, 不了解新观念、新方法, 不参与 实际的物流活动, 必然产生“强理论, 弱实践”的现象，使理论与实践脱节，无法圆满实现该 课程的教学目标及物流管理专业人才培养目标。

\section{3 新技术使用缺乏, 教学内容创新太少}

仓储管理是一门实践性很强的课程, 学生的综合应用能力是培养的主要目标, 因此在进行 课程考核时, 应突出对实践操作能力的考核, 如实训环节、实验环节等。然而由于缺乏新技 术, 无法进行复杂理论的展示和模拟, 导致学生在进行实验教学中缺乏主动性和创造性, 仅 仅是简单的模仿和模拟。

\section{2. 引入虚拟现实技术提升实验教学模式的改革}

\section{1 虚拟现实技术的技术特点}

1) 感知性。感知性主要通过环境再现来实现, 感知性是虚拟现实技术的一个重要特征。

2) 交互性。交互性可以通过设定预期操作结果, 可以在虚拟现实环境中进行模拟, 并通过 对设备的实践应用来达到预期的目的，实现设备的交互操作。

3) 沉浸性。沉浸性主要是针对体验者的个人感知而言, 由于虚拟现实技术所创造的环境与 真实环境相仿, 而体验者在潜意识里仍然意识到这是仿真非真实环境。

4) 客观性。客观性更多的是指仿真环境中的物品或者营造的氛围都按照现实中进行仿造, 其仿造技术与现实大体相似, 物品和设备的排列方式和运动方式也与现实大体吻合, 其物体 运动也符合现实中的物理定律, 从客观角度对现实环境进行模仿。

\section{2 虚拟现实对实验教学的促进意义}

1) 场景的虚拟性。在虚拟实验中操作的所有流程都是依托教学内容, 进行有针对性模拟, 从而实现教学内容真实感, 有助于解决实验项目空洞, 解决实验空间和实验成本, 改善实验 条件。

2) 实验内容的多目标、多层次性。根据虚拟实验环境针对具体的实验内容提出针对性的实 验要求, 并能满足不同类型实验的需求, 因此虚拟实验内容的多目标、多层次性有助于学生 把专业的知识与实验内容结合起来, 有效地发挥自身的能动性, 借助于实验方式更好地理解 理论知识, 为后续的专业学习提高应对。

3) 实验手段的灵活性, 主要表现为: 在虚拟实验过程中, 学生能方便地改变事物的条件以 观察所发生的变化, 有利于学生获得第一手资料, 便于学生根据自己的假设分析实验数据。 实验的体验感更好, 更能激发学生进一步提出问题与寻求解决问题的途径。

\section{3. 基于虚拟现实技术的仓储管理实验教学新模式}

以湖北经济学院仓储管理实验教学为例, 我校以 simpliquity 虚拟场景, 搭建了仓库组织、 订单拣选、ABC 分类、补货、货架存储等模块, 旨在借助虚拟现实来模拟仓储管理理论课程, 对仓储物流系统进行合理系统规划、调度方案以及流程优化等实验模拟工作，有效提升学生 在仓储管理实验教学的兴趣, 可以根据学习的效果进行内容的演示和验证, 有助于虚实结合。

\section{1 以虚拟现实对 $\mathrm{ABC}$ 理论进行模拟, 优化库存商品}

实验步骤一: 按照课程要求设计仓库布局, 结合所做的调研数据分析每种货品出入库频率。 
实验步骤二：按照虚拟仿真场景设计

1) 放置实验页 “ABC 分析”，实验系统显示空白仓库;

2) 在仓库中摆放 14 个货架（每个货架有 9 个托盘位，14 个货架可以达到 $14 * 9=126$ ），1 个办公室和出入库台;

3) 实验系统判断货架和出入库区域的间距是否符合叉车的通行要求, 如果不满足要求, 实 验系统会出现红色警报; 如果满足叉车通行要求, 实验系统显示绿色通行通道; 如果货架上 托盘的影像闪炼或者没有托盘显示, 说明该货架没有被实验系统识别, 需要调整货架位置直 到系统稳定 (不能稳定识别的货架, 在模拟运行时, 系统认为该处不存在货架, 会在此处生 产叉车通道)。

4) 完成仓库布局设计, 在实验指导书的模拟“+”上放置黑白按钮, 在速度上放置黑白按钮, 并向右侧移动该按钮, 调整模拟运行速度为最大值 $20 \mathrm{x}$ 。

5) 运行模型，记录仓库搬运 1000 个托盘的时间和平均每小时拣选托盘的数量;

6) 调整仓库布局, 重复 3 5 步骤, 比较不同仓库布局对出入库效率。

7) 选择比较好的布局, 模拟运行, 记录每个 SKU 的拣选数量。

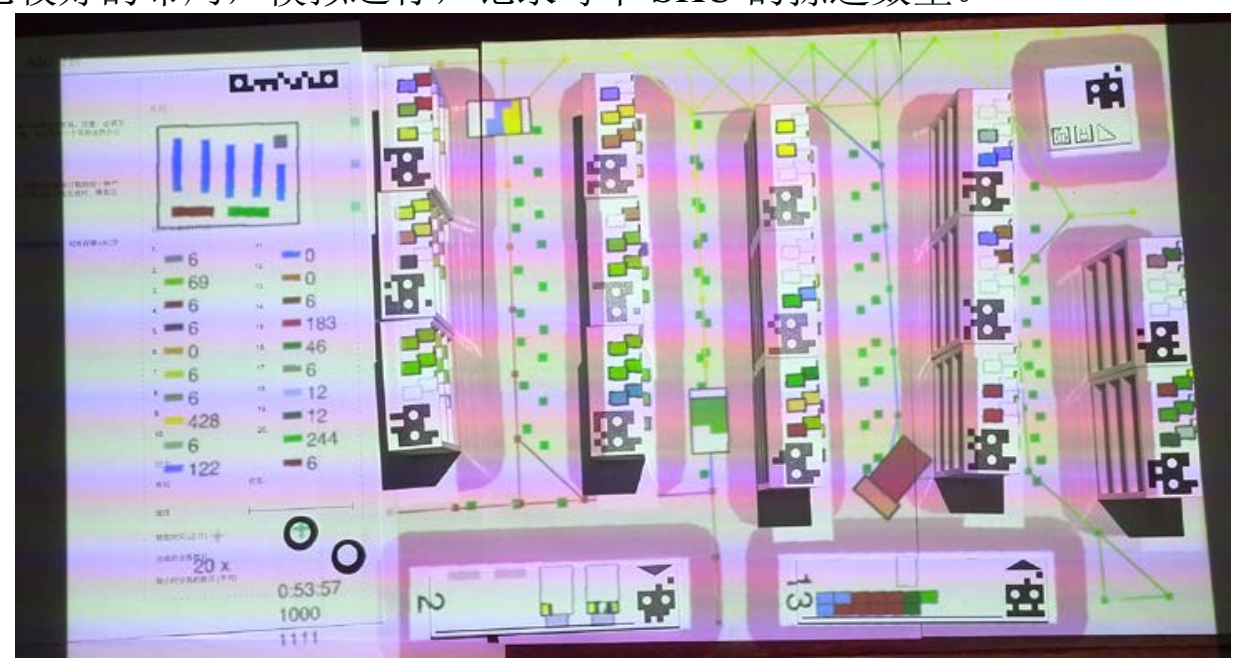

这种基于虚拟现实技术的仓储管理实验教学模拟对于现有的传统实验教学是一种方式的 提升, 有助于打破理论教学和实际操作之间的屏障, 提高学生动手能力。根据以前的教学经 验, 学生对于仓储流程这方面的内容大部分处于理论认识阶段, 通过课堂学习之后如果缺少 定期强化, 则一部分关键知识将会被遗忘; 有助于加深学生对专业重点知识的认识和了解, 学生能自主对仓储的布局、运输路线、货物的承载量、运输工具的匹配等进行模拟, 加深了 对仓储流程知识的理解和印象, 从而达到较为理想的教学效果; 有助于促进实验教学与课堂 教学的紧密结合。该项目将积极收集学生对实验操作的体会, 并根据学生的具体需求, 对实 验内容及实验指导书进行不断修正和完善, 从而促进实验教学及课堂教学的紧密结合。

\section{2 围绕虚拟现实技术在实验教学体系的优化}

在虚拟现实技术的课程结构体系的指导下, 依据《仓储管理》课程的教学目标和培养目标, 对课程教学体系进行优化, 全面将新技术引入作为实验教学培养的基本能力, 不断丰富性技 术的运用能力, 主要包括以下两个方面:一是实验课程教学体系的优化。内容的设计可以依托 物流企业调研和横向课题, 把解决题作为教学方式的创新点, 利用虚拟现实技术进行模拟, 保证教学内容能够反映最新理论和应用成果。二是课程实验教学体系的融合。实验课程项目 应与物流的实际发展和社会需求相融合, 开发具有时代性和先进性的实验内容项目, 形成从 低到高、由浅及深, 包括基础验证性、综合设计性和研究创新性等在内的层次多样、系统合 理的实验教学体系。 


\section{4. 综述}

在实验教学环节中通过逼真的虚拟环境实现仓储管理理论演示，搭建以仓库组织、订单拣 选、ABC 分类、补货、货架存储等模块, 突破了传统的教学模式, 在仓储管理专业实验教学 中应用虚拟现实技术手段, 可以根据教学需要自由搭建功能模块, 让学生在仓储管理实验中 交互式学习, 由传统的被动学习转化我要学, 学以致用能力学习, 能大大提高实验教学效果。 同时也积极与国内外软件公司合作, 开发更多的仓储管理模拟模块, 增加应用场景, 可使虚 拟现实技术在高校物流实践教学发挥更多作用。

\section{参考文献}

[1] 许婧.VR 技术在物流工程专业实践教学中的应用研究 [J]. 物流工程与管 理,2016(6):279-280;

[2] 邱 健. 仓储系统建模与仿真[J]。科学技术通讯, 2008, (5) :26-29;

[3] 宋建新, 徐菱, 宋远卓. 现代生产物流系统仿真研究 $[\mathrm{J}]$. 物流科技, 2007( 5) :32;

[4] 陈榕. 虚拟现实技术在物流园区规划中的应用 [J]．物流科技，2012(7):71-72;

[5] 翟晓松.基于互联网+VR 引领下的物流管理专业课程教学改革实践应用研究 $[\mathrm{J}]$.物流工程 与管理,2018(5):163-165;

[6] 张鹏举, 陈昆昌等.VR 仿真平台在自动化仓储系统中的设计与实现[J].计算机工程, 2008 (11) : 250-252。 